\title{
Cumplimiento de las Recomendaciones de la Guía SEH/ SEC 2007 en población Hipertensa en un Centro de Salud
}

\author{
Ignacio M. Sánchez Barrancos ${ }^{a}$, Pedro Domínguez Sánchez-Migallón ${ }^{\mathrm{b}}$, Domingo Zamora Martín ${ }^{\mathrm{b}}$, \\ Antonio Iglesias Fernándeza, Rafael García García ${ }^{b}$, Francisco Alhambra García Cervigóna, \\ Ana María Román Ruiz del Moral ${ }^{b}$, Carmen Martínez Gallardoa, Antonio del Valle Cuervaa
}

\begin{abstract}
${ }^{a}$ Consultorio de Membrilla (Centro de Salud Manzanares II, Manzanares, Ciudad Real).

${ }^{b}$ Centro de Salud MANZANARES II, (Manzanares, Ciudad Real).
\end{abstract}

\section{Correspondencia:} Ignacio M Sánchez Barrancos, Consultorio de Membrilla, c/ Lonja, s/n, 13230 Membrilla (Ciudad Real, España). Tfnos: 926637873 / 607670015, e-mail: imanuels@jccm.sescam.es, ignaciomsb@telefonica.net

Recibido el 16 de febrero de 2009.

Aceptado para su publicación el 13 de marzo de 2009.

\section{RESUMEN}

Objetivo. Evaluar la proporción de hipertensos con y sin factores de riesgo cardiovascular añadido que tienen presión arterial controlada, y el seguimiento de la Guía SEH/SEC 2007 por los médicos de un Centro de Salud.

Diseño. Estudio observacional descriptivo transversal.

Emplazamiento. Zona de Salud Manzanares II.

Participantes. Pacientes adultos con Hipertensión Arterial documentada en la historia clínica informatizada, reclutados mediante muestreo sistemático.

Mediciones. Antecedentes familiares y personales de factores de riesgo cardiovascular, biometría (edad, sexo, peso, talla, índice de masa corporal), presión arterial, datos analíticos, electrocardiograma y fármacos empelados.

Resultados. La proporción de pacientes hipertensos con cifras controladas $(<140 / 90 \mathrm{mmHg})$ fue de 50,35\% (IC 95\%: 48,17-52,54\%), siendo significativamente inferior en hombres (47,46\%, IC 95\%: $44,03-50,89)$ que en mujeres (52,44\%, IC 95\%: 49,60-55,28\%) ( $p=0,01)$. La proporción de controlados entre los que tienen riesgo añadido fue mayor $(56,52 \pm 14,40 \%)$ que la de aquéllos sin riesgo añadido $(49,15 \pm 6,41 \%)$, sin diferencias entre sexos. El $36,86 \%$ de los pacientes con riesgo tomaba estatinas y, de ellos, el 60,92\% tenía LDL > $130 \mathrm{mgr} / \mathrm{dl}$. El 32,05\% de los hipertensos diabéticos tomaba estatina y antiagregante y, de éstos, el $40 \%$ tenía $\mathrm{LDL}<100 \mathrm{mgr} / \mathrm{dl}$ y el $24,36 \% \mathrm{HBA} 1 \mathrm{C}<$ $6,5 \%$.

Conclusiones. La proporción de hipertensos con cifras de presión arterial controladas fue elevada en relación a otros estudios de nuestro ámbito. Debemos mejorar el seguimiento de la Guía SEH/ SEC para el abordaje integral y la calidad de la asistencia a nuestros hipertensos.

Palabras clave. Hipertensión, Cooperación del Paciente, Factores de Riesgo.

\section{ABSTRACT}

Compliance to the 2007 ESH/ESC Guideline in a hypertensive population at a Primary Health Care Centre

Objective. To determine the proportion of controlled hypertensive patients with and without added cardiovascular risks factors and to evaluate compliance to the 2007 SHE/SEC guidelines by doctors at a Primary Health Care Centre.

Design. Observational, descriptive, cross-sectional study.

Setting. Primary Health Care Centre at Manzanares II (Ciudad Real).

Subjets. Adult patients with documented hypertension in their computerised medical record. Patients were recruited by systematic sampling

Main measurement. Family and personal history of cardiovascular risk factors, biometric (age, sex, weight, height, body mass index), blood pressure, analytical data, electrocardiogram and medication.

Results. The percentage of patients with controlled hypertension $(<140 / 90 \mathrm{~mm} \mathrm{Hg})$ was $50.35 \%$ (48.17 -52.54\%) being lower in males $47.46 \%$ (44.03 - 50.89\%) than in females 52.44 (49.6-55.28\%) with statistical significance $(\mathrm{p}=0.01)$. The percentage of well controlled patients with added risks factor was greater $(56.52+/-14.4 \%)$ than those without added risk factors $(49.15+/-6.41 \%)$, with no differences between sexes. $36.86 \%$ of the patients with risk factors were taking statins and of this group $60.92 \%$ had LDL levels of $>130 \mathrm{mg} / \mathrm{dl}$. $32.05 \%$ of the diabetic patients with high blood pressure were taking statins and antiaplatelets and $40 \%$ of them had LDL $<100 \mathrm{mgr} / \mathrm{dl}$ and $24.36 \%$ had HBA1C $<6.5 \%$.

Conclusions: The percentage of patients with controlled hypertension was high compared to other studies in the same setting. We should improve compliance to the ESH/ESC guidelines in order to provide complete, high quality care to our hypertensive patients.

Key words: Hypertension, Patient Compliance, Risk Factors. 


\section{INTRODUCCIÓN}

La enfermedad cardiovascular (ECV), y especialmente la enfermedad coronaria, sigue constituyendo la primera causa de mortalidad en los países desarrollados. Dada la heterogeneidad de procesos y la multiplicidad de alternativas preventivas y terapéuticas, la variabilidad de las actuaciones de los profesionales sanitarios frente al manejo de la ECV y la identificación y abordaje de los factores de riesgo cardiovascular (FRCV) es norma común. La evidencia disponible es recogida en las guías elaboradas por grupos de trabajo de expertos ${ }^{1}$, que vienen a establecer los procedimientos de diagnóstico, control y seguimiento más acordes con los conocimientos actuales, constituyéndose en una valiosa herramienta para disminuir esa variabilidad.

En la actualidad, un aspecto esencial recogido en todas las guías para el abordaje de la Hipertensión Arterial (HTA), derivado de la frecuencia con que ésta se asocia a los demás FRCV y a la afectación subclínica de órganos, es que se aconseja clasificar a los pacientes hipertensos no sólo en relación al grado de la HTA, sino también en relación al riesgo cardiovascular total debido a la concomitancia de diferentes FRCV, lesiones de órganos y enfermedades ${ }^{1,2,5,9-11}$. De acuerdo con las recomendaciones de la Guía de la Sociedad Europea de Hipertensión (SEH) y la Sociedad Europea de Cardiología (ESC)1, en los pacientes hipertensos el objetivo principal del tratamiento es alcanzar una reducción máxima del riesgo total de ECV a largo plazo, lo cual obliga a tratar tanto la HTA como todos los FRCV asociados. Sin embargo, a pesar de una evidencia científica cada vez mayor, la investigación demuestra que el conocimiento y el seguimiento de las guías de prevención cardiovascular y sus recomendaciones es muy escaso por parte de los médicos de familia y los distintos especialistas implicados en la atención sanitaria de los pacientes con ECV y/o sus FRCV $2,3,4$.

El médico de familia, por su privilegiada ubicación en el sistema sanitario y por su conocimiento intensivo y extensivo de los pacientes, debe constituirse en un pilar fundamental para la detección, la modificación y el tratamiento de los FRCV en la población general, en aras de establecer estrategias terapéuticas que logren la consecución de los objetivos recomendados en las guías (MEV, reducción de PA, intervención y control sobre el riesgo cardiovascular total), y reducir la morbimortalidad por ECV. Una mayor adhesión del médico de familia a las recomendaciones de las guías actuales se invoca como mecanismo para alcanzar este objetivo ${ }^{1-3,6,7}$.

Nos planteamos en este estudio intentar contestar a la siguiente cuestión: ¿en qué grado conseguimos en el Centro de Salud Manzanares II los objetivos recomendados actualmente por la guía SEH/SEC 2007 en nuestros hipertensos? Para ello establecimos, como objetivo principal, conocer el grado de control de la PA en los pacientes hipertensos con y sin riesgo añadido, y como objetivos secundarios, conocer el porcentaje de pacientes hipertensos con riesgo añadido que están tratados con estatinas, el porcentaje de pacientes hipertensos en tratamiento con estatina con niveles de LDL en el objetivo terapéutico, el porcentaje de pacientes hipertensos con diabetes tipo 2 que tienen tratamiento con estatina y antiagregantes y el porcentaje de pacientes hipertensos con diabetes tipo 2 que tienen un adecuado control metabólico.

\section{MATERIAL Y MÉTODO}

Estudio observacional descriptivo transversal en el ámbito del E.A.P. Manzanares II (que incluye la población de Membrilla y más del $50 \%$ de la población de Manzanares) con la participación de todos los cupos médicos, siendo susceptibles de ser incluidos en el estudio todos los pacientes hipertensos de la Zona de Salud, identificados mediante listado específico obtenido a través de Turriano. Los criterios de inclusión fueron: 1) pacientes con HTA documentada en la historia clínica (diagnosticados con tres tomas diferentes sucesivas con promedio igual o superior a $140 / 90 \mathrm{mmHg}$ ), 2) pacientes con diagnóstico previo de HTA en el momento de abrir la historia, 3) pacientes en cuya historia existía referencia expresa al control, tratamiento o seguimiento de la HTA, aunque no constase como tal el diagnóstico y/o la pertenencia al grupo de riesgo y/o la inclusión en el respectivo protocolo. Los criterios de exclusión fueron: 1) no pertenecer realmente al cupo (desplazado y/o adscripciones temporales) aún figurando en el listado de hipertensos, 2) pacientes en cuya historia clínica no constase la información requerida en la hoja de recogida de datos (HRD) (en este supuesto fue sustituido por el inmediato siguiente en el listado de hipertensos).

Para el cálculo del tamaño muestral se empleó la fórmula de proporciones en poblaciones finitas, donde $p=0,29^{15}$, con una confianza del $95 \%$ y un error muestral (alfa) del 5\%. La selección de los 282 pacientes (118 hombres y 164 mujeres) se realizó mediante muestreo sistemático sobre el listado de hipertensos de toda la Zona de Salud. Cada médico participante cumplimentó una HRD por cada paciente seleccionado, anotando los datos más recientes posibles y aceptando como rango máximo de validez: 12 meses para datos analíticos, 6 meses para peso e IMC (excepto en inmovilizados) y cifras tensionales en rang de control, 3 meses para cifras tensionales fuera de rango de control y 2 meses para todos los fármacos relacionados con la TA y enfermedades concomitantes. Se consideraron como cifras de control de PA las establecidas en la Guía SEC/SEH 2007.

El análisis estadístico se realizó con el programa GSTAT 2, empleando la comparación de medias mediante la "t" de Student y la prueba Z para la comparación de proporciones. La extrapolación a la totalidad de nuestros hipertensos se realizó con el cálculo de los intervalos de confianza (IC 95\%). 


\section{RESULTADOS}

La población total mayor de 14 años de la Zona de Salud es de 14.161 habitantes, 6.829 hombres $(48,2 \%)$ y 7.332 mujeres $(51,8 \%)$, de los cuales tienen diagnóstico de HTA 2.026 individuos (824 hombres y 1202 mujeres). De ello resulta una prevalencia de hipertensión diagnosticada de 14,37\% (IC95\%: 13,8$14,96)$, mayor entre las mujeres $(16,39 \%$; IC 95\%: $15,55-17,26)$ que entre los hombres $(12,07 \%$; IC 95\%: 11,3-12,86) con una diferencia estadísticamente significativa $(p<0,005)$.

Los valores medios de presión arterial (PA) fueron de 136,82 mmHg $(134,9-138,6 \mathrm{mmHg})$ para la PA sistólica (PAS) y de 78,6 mmHg $(77,5-79,7 \mathrm{mmHg})$ para al PA diastólica (PAD), y la frecuencia cardiaca media fue de 73,8 latidos por minuto $(72,7-74,9)$, sin existir diferencias por sexo en los pacientes (total e HTA controlada) (tabla 1 y figura 1).

La proporción de pacientes hipertensos con cifras de PA controladas (inferiores a $140 \mathrm{mmHg}$ de PAS y 90 $\mathrm{mmHg}$ de PAD) fue del 50,35\% (IC 95\%: 48,17-52,54), siendo inferior la proporción de hombres controlados

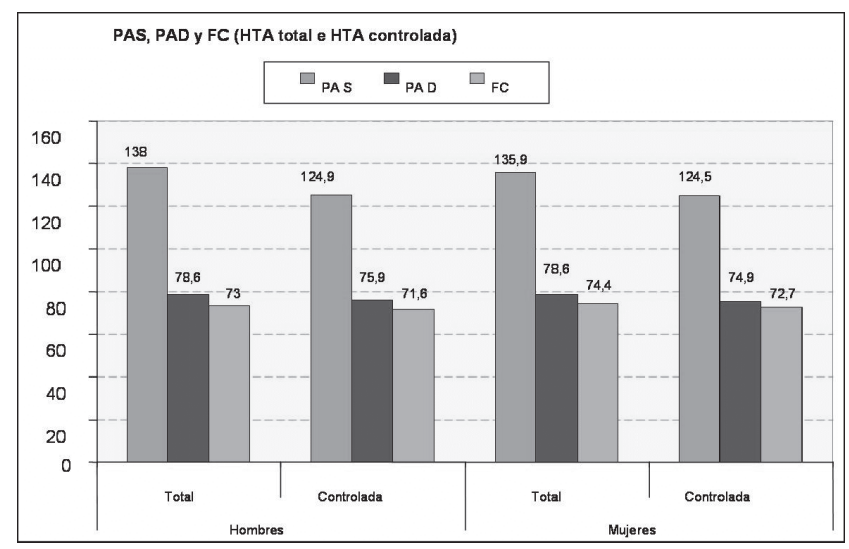

Figura 1. Cifras tensionales.

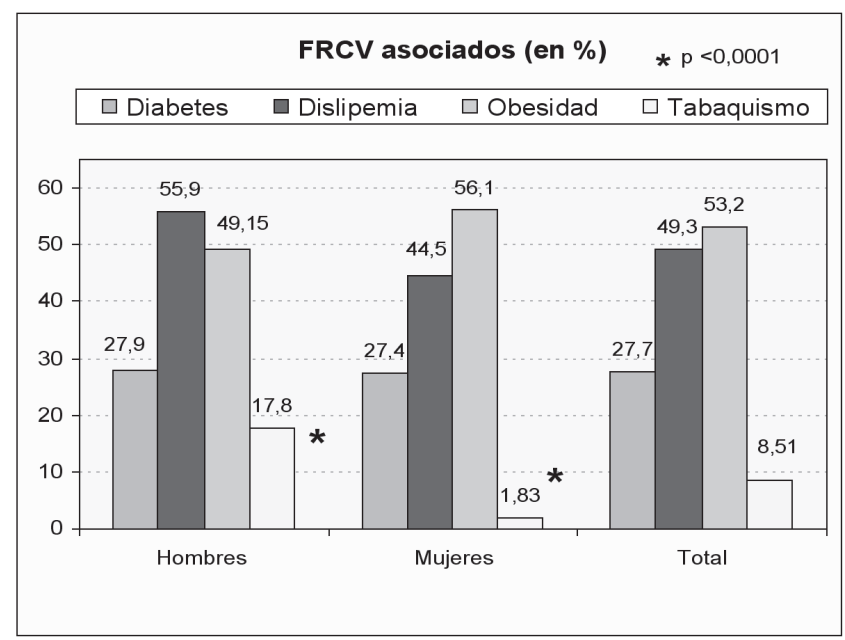

Figura 2. FRCV asociados.
(47,46\%; IC 95\%: 44,03-50,89) que la de mujeres controladas (52,44\%; IC 95\%: 49,6-55,28\%), con una diferencia estadísticamente significativa (4,98\%; IC 95\%: 0,55-9,41; $p=0,01$ ) (tabla 2).

La proporción de hipertensos con riesgo CV añadido y cifras de PA controladas fue mayor $(56,52 \pm 14,4 \%)$ que la de aquéllos sin riesgo CV añadido (49,15 \pm $6,41 \%)$, sin obtenerse diferencias entre sexos en ambos casos (tabla 2).

Entre los factores de riesgo asociados a HTA encontrados en nuestros pacientes destacan por su frecuencia obesidad, dislipemia, diabetes y tabaquismo (53,2\%, 49,3\%, 27,7\% y 8,51\%, respectivamente) (figura 2) sin diferencias por sexos, excepto para tabaquismo ( $p<0,0001)$. El número medio de factores de riesgo fue significativamente superior $(p=0,004)$ en hombres (1,98; IC 95\%: 1,75-2.21) que en mujeres $(1,48$; IC 95\%: 1,32-1,65) (tabla 1).

La existencia de enfermedades clínicas aisladas (cardiopatía isquémica, ictus, enfermedad arterial periférica y/o enfermedad renal) presentó una prevalencia variable (con diferencias entre sexos en la muestra total) (tabla 1), no apareciendo diferencias en los pacientes con cifras controladas.

En cuanto a los datos de laboratorio, los valores medios de los parámetros lipídicos de nuestra población hipertensa (total y con TA controlada) se muestran en la figura 3, obteniendo diferencias significativas $(p<0,005)$ por sexo en los valores medios de colesterol HDL. En cuanto a los niveles de creatinina plasmática (0,23 mg/dl mayor en hombres, IC 95\%: 0,16-0,29) y microalbuminuria $(22,74 \mathrm{mg}$ superior en hombres, IC 95\%: 2-42) (tabla 1) existieron diferencias entre sexos en la muestra total. Destaca que en los pacientes con las cifras tensionales controladas no hubo diferencias en la presencia de microalbuminuria, manteniéndose las diferencias entre sexos en las cifras de creatinina.

A un $76,2 \%$ de los pacientes $(70,8-81,1 \%)$, sin diferencias significativas entre sexos (tabla 1), se les había realizado un EKG en los dos últimos años de seguimiento, y de ellos, el 10,5\% (6,72-15,5\%), igualmente sin diferencia entre sexos, cumplían el criterio de Cornell para el diagnostico de HVI (tabla 1).

En el tratamiento concomitante (no antihipertensivo) de nuestros pacientes hipertensos (total y controlados) no hemos hallado diferencias por sexos (figura 4), excepto para el empleo de fármacos antiagregantes en la población hipertensa total, mayor en varones $(49,15 \pm 9,67 \%)$ que en mujeres $(33,54 \pm 7,18 \%)$, siendo la diferencia estadísticamente significativa $(\mathrm{p}<$ 0,05) (15,61\%; IC 95\%: 2,56-33,58) (tabla 1).

En cuanto al tratamiento farmacológico antihipertensivo, nuestros pacientes tomaban un número medio de fármacos hipotensores de 1,73 fármacos (IC95\%: $1,62-1,84)(1,83$ en hombres y 1,65 en mujeres), sin 


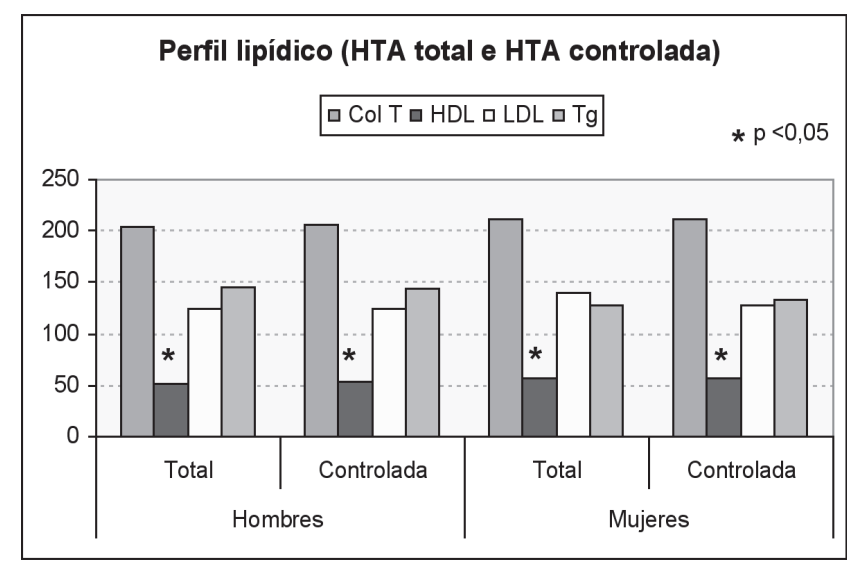

Figura 3. Perfil lipídico.

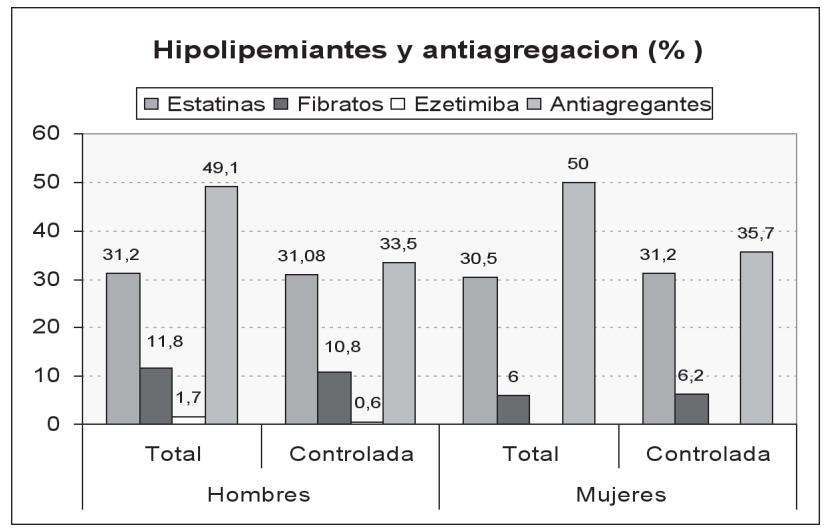

Figura 4. Uso de fármacos no antihipertensivos.

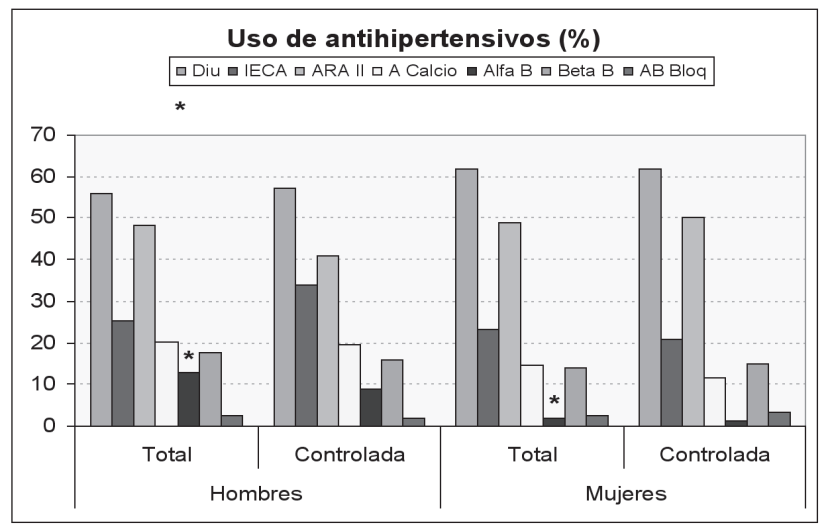

Figura 5. Uso de antihipertensivos.

diferencias entre sexos (tabla 1). El porcentaje de antihipertensivos es el reflejado en la figura 5, destacando el amplio uso de diuréticos $(59,22 \pm 5,79 \%)$, lo cual es debido al uso de tratamiento combinado de diuréticos con ARA II y/o IECA, si bien se usan en monoterapia en el 11,35\% (IC 95\%: 7,8-11,5). Existieron diferencias ( $p=0,002)$ entre sexos en el uso de alfabloqueantes (en hombres 12,71\%; IC 95\%: 7,3-20,1 y en mujeres 1,82\%; IC 95\%: 0,3-5).

La proporción de hipertensos con riesgo CV añadido que tomaban estatinas fue del $36,86 \% \pm 0,18 \%$, sin diferencias entre sexos (tabla 2). De éstos, el 60,92\% $\pm 10,3 \%$ tenían cifras de colesterol LDL en niveles objetivo (menor o igual a $130 \mathrm{mg} / \mathrm{dl}$ ), encontrando este porcentaje mayor en hombres $(70,27 \% \pm 14,8 \%)$ que en mujeres $(54,0 \% \pm 13,89 \%)$ (tabla 2$)$, próximo a la significación estadística $(p=0,06)$.

El 32,05\% (13,66-50,44\%) de los hipertensos diabéticos tomaba estatina más antiagregante plaquetario, y de éstos sólo el 40,0\% (9,48-70,52\%) presentaba niveles de colesterol LDL en cifras objetivo $(<100$ $\mathrm{mg} / \mathrm{dl})$, representando éstos únicamente el 12,82\% (5,36-20,28\%) del total de hipertensos diabéticos. No hemos hallado diferencias por sexos (tabla 2).

El porcentaje hallado de hipertensos diabéticos con control metabólico adecuado es escaso, en función del criterio de corte elegido: hemoglobina glicosilada inferior a 7\% (35,90\%; IC95\%: 18,04-53,76) o hemoglobina glicosilada inferior a 6,5\% (24,36\%; IC 95\%: 4,96-43,76\%).

En el grupo de pacientes con cifras de presión arterial controladas se analizaron las mismas variables que en la muestra, habiéndose comprobado que, de forma similar a la población hipertensa de la que provienen, existen diferencias entre ambos sexos en el número de FRCV y en las cifras de creatinina, pero no en los valores de microalbuminuria (tabla 1).

Por otro lado, destacan como deficiencias:

- A 6 pacientes de nuestra muestra no se les había realizado nunca un perfil lipídico, ni una determinación de creatinina, lo que nos permite estimar esa deficiencia en el $2,12 \%(0,78-4,57 \%)$ de nuestros hipertensos.

- A 29 pacientes no se les había realizado nunca un cálculo de filtrado glomerular, lo que permite estimar el déficit en $12,06 \%(7,5-16,63 \%)$.

- A 67 pacientes no se les había realizado nunca un ECG, lo que permite estimar el déficit en $23,76 \%$ (18,91-29,17\%) de nuestros hipertensos.

- A 151 pacientes no se les había realizado nunca una determinación de microalbuminuria en orina, lo que permite estimar el déficit en el 53,54\% (48-59,1\%).

\section{DISCUSIÓN}

Hemos hallado una proporción de pacientes controlados elevada en relación con los datos publicados en nuestro ámbito ${ }^{13,14,15}$, lo que ponemos en relación con lo siguiente:

- El diseño del estudio permite estimar el grado de control de las cifras de presión arterial en hipertensos registrados con historia clínica informatizada. Es posible que algunos hipertensos ya diagnosticados no estén adecuadamente registrados, dada la desigual implementación de los registros entre los distintos profesionales médicos y de enfermería del equipo, 


\begin{tabular}{|c|c|c|c|c|c|c|}
\hline \multirow{2}{*}{$\frac{\text { Parámetro/s }}{\text { Cifras tensionales }}$} & \multicolumn{2}{|c|}{ Total Muestra (IC 95\%) } & \multicolumn{4}{|c|}{ HTA controlada (IC 95\%) } \\
\hline & Hombres $(n=118)$ & Mujeres (n=164) & $\mathbf{P}$ & Hombres $(n=56)$ & Mujeres ( $n=86$ ) & $\mathbf{P}$ \\
\hline P Arterial Sistólica & $\begin{array}{c}138,0 \\
(135,0-140,9)\end{array}$ & $\begin{array}{c}135,9 \\
(133,6-138,2)\end{array}$ & NS & $\begin{array}{c}124,9 \\
(122,4-126,0)\end{array}$ & $\begin{array}{c}124,5 \\
(122,9-126,0)\end{array}$ & NS \\
\hline P Arterial Diastólica & $\begin{array}{c}78,5 \\
(76,9-80,2)\end{array}$ & $\begin{array}{c}78,6 \\
(77,2-80,1)\end{array}$ & NS & $\begin{array}{c}75,9 \\
(74,3-77,6)\end{array}$ & $\begin{array}{c}74,9 \\
(73,4-76,4)\end{array}$ & NS \\
\hline Frecuencia Cardiaca & $\begin{array}{c}73,0 \\
(71,3-74,6)\end{array}$ & $\begin{array}{c}74,4 \\
(72,8-75,9)\end{array}$ & NS & $\begin{array}{c}71,6 \\
(69,4-73,6)\end{array}$ & $\begin{array}{c}72,7 \\
(70,7-74,8)\end{array}$ & $\begin{array}{l}\text { NS } \\
\text { NS }\end{array}$ \\
\hline A. F. de ECV precoz & $\begin{array}{c}3 \\
(2,54 \%)(0,5-7,2)\end{array}$ & $\begin{array}{c}6 \\
(3,6 \%)(1,3-7,7)\end{array}$ & NS & $\begin{array}{c}2 \\
(3,57 \%)(0,4-12,3)\end{array}$ & $\begin{array}{c}3 \\
(3,49 \%)(0,7-9,8)\end{array}$ & $\begin{array}{l}\text { NS } \\
\text { NS }\end{array}$ \\
\hline \multicolumn{7}{|l|}{ A. Personales de ECA } \\
\hline C. Isquémica & $\begin{array}{c}(n=24) 20,34 \% \\
(13,5-28,7)\end{array}$ & $\begin{array}{c}(n=12) 7,32 \% \\
(3,8-12,4)\end{array}$ & 0,0012 & $\begin{array}{c}(n=13) 23,2 \% \\
(12,9-36,4)\end{array}$ & $\begin{array}{c}(n=11) 12,79 \% \\
(6,5-21,3)\end{array}$ & NS \\
\hline ACVA & $\begin{array}{c}(n=10) 8,47 \% \\
(4,1-15,0)\end{array}$ & $\begin{array}{c}(n=5) 3,05 \% \\
(1,0-6,9 \%)\end{array}$ & 0,045 & $\begin{array}{c}(n=4) 7,14 \% \\
(1,9-17,2)\end{array}$ & $\begin{array}{c}(0,73-9,86) \\
0\end{array}$ & NS \\
\hline E Arterial Periferica & $\begin{array}{c}(n=6) 5,08 \% \\
(1,9-10,7)\end{array}$ & 0 & & $\begin{array}{c}(n=3) 5,36 \% \\
(1,1-14,8)\end{array}$ & & \\
\hline Enfermedad renal & $\begin{array}{c}(n=14) 11,86 \% \\
(6,6-19,1)\end{array}$ & $\begin{array}{c}(n=8) 4,88 \% \\
(2,1-9,4)\end{array}$ & 0,03 & $\begin{array}{c}(n=6) 10,71 \% \\
(4,0-21,8)\end{array}$ & $\begin{array}{c}(n=5) 5,81 \% \\
(1,9-13,0)\end{array}$ & NS \\
\hline FRCV (número) & $\begin{array}{c}1,98 \\
(1,75-2.21)\end{array}$ & $\begin{array}{c}1,48 \\
(1,32-1,65)\end{array}$ & 0,0004 & $\begin{array}{c}1,91 \\
(1,55-2,26)\end{array}$ & $\begin{array}{c}1,48 \\
(1,32-1,72)\end{array}$ & $0 ’ 04$ \\
\hline \multicolumn{7}{|l|}{ Datos analíticos } \\
\hline Glucemia (mg/dl) & $\begin{array}{c}116,5 \\
(110,7-122,3)\end{array}$ & $\begin{array}{c}115,9 \\
(110,0-121,7)\end{array}$ & NS & $\begin{array}{c}111,6 \\
(104,5-118,7)\end{array}$ & $\begin{array}{c}110,2 \\
(104,2-116,3)\end{array}$ & NS \\
\hline Creatinina (mg/dl) & $\begin{array}{c}1,04 \\
(0,98-1,09)\end{array}$ & $\begin{array}{c}0,81 \\
(0,77-0,84)\end{array}$ & 0,002 & $\begin{array}{c}1,01 \\
(0,94-1,09)\end{array}$ & $\begin{array}{c}0,81 \\
(0,76-0,86)\end{array}$ & $<0,0001$ \\
\hline MAU (mg) & $\begin{array}{c}32,15 \\
(8,2-56,0)\end{array}$ & $\begin{array}{c}9,41 \\
(4,06-14,7)\end{array}$ & 0,003 & $\begin{array}{c}14,77 \\
(3,2-26,3)\end{array}$ & $\begin{array}{c}10,51 \\
(1,4-19,5)\end{array}$ & NS \\
\hline \multicolumn{7}{|l|}{ Otras exploraciones } \\
\hline ECG (\%) & $\begin{array}{c}82,2 \\
(74,0-88,6)\end{array}$ & $\begin{array}{c}71,9 \\
(64,4-78,6)\end{array}$ & NS & $\begin{array}{c}80,3 \\
(67,5-89,6)\end{array}$ & $\begin{array}{c}74,4 \\
(63,8-83,2)\end{array}$ & NS \\
\hline Existencia de HVI (\%) & 11,9 & 9,4 & NS & 15,9 & 7,81 & NS \\
\hline & $(6,12-20,3)$ & $(4,7-16,2)$ & & $(6,64-30,7)$ & $(2,59-17,3)$ & \\
\hline $\begin{array}{l}\mathrm{N}^{\circ} \text { de fármacos } \\
\text { anti-hipertensivos }\end{array}$ & $\begin{array}{c}1,83 \\
(1,65-2,02)\end{array}$ & $\begin{array}{c}1,65 \\
(1,53-1,78)\end{array}$ & NS & $\begin{array}{c}1,78 \\
(1,5-2,06)\end{array}$ & $\begin{array}{c}1,62 \\
(1,46-1,78)\end{array}$ & NS \\
\hline \multicolumn{7}{|l|}{ Otros fármacos } \\
\hline Antiagregantes (\%) & $\begin{array}{c}49,1 \\
(39,8-59,8)\end{array}$ & $\begin{array}{c}33,5 \\
26,3-41,3)\end{array}$ & 0,008 & $\begin{array}{c}55,3 \\
(31,3-58,5)\end{array}$ & $\begin{array}{c}41,8 \\
(31,3-52,9)\end{array}$ & NS \\
\hline
\end{tabular}

Tabla 1. Resumen de datos más relevantes. NS: No Significativo ( $p \geq 0,05)$; ECV: Enfermedad Cardiovascular; ECA: Enfermedad Clínica Asociada, MAU: Microalbuminuria (1 muestra); HVl: Hipertrofia Ventricular izquierda (Criterios de Cornell).

\begin{tabular}{|c|c|c|c|c|}
\hline & $\begin{array}{l}\text { Hombres } \\
\text { Mujeres }\end{array}$ & $\begin{array}{l}\text { (IC 95\%) } \\
\text { (IC 95\%) }\end{array}$ & $\begin{array}{c}\text { Total } \\
\text { (IC 95\%) }\end{array}$ & $\mathrm{p}$ \\
\hline Grado de control de PA (total) & $\begin{array}{c}47,46 \% \\
(44,03-50,88)\end{array}$ & $\begin{array}{c}52,44 \% \\
(49,6-55,28)\end{array}$ & $\begin{array}{c}50,35 \% \\
(48,17-52,54)\end{array}$ & 0,01 \\
\hline Grado de control con RCV añadido & $\begin{array}{c}58,82 \% \\
(35,31-82,34 \%)\end{array}$ & $\begin{array}{c}55,17 \% \\
(36,98-73,37)\end{array}$ & $\begin{array}{c}56,52 \% \\
(42,12-70,92)\end{array}$ & NS \\
\hline Grado de control sin RCV añadido & $\begin{array}{c}45,54 \% \\
(35,78-55,31)\end{array}$ & $\begin{array}{c}51,85 \% \\
(43,38-60,32)\end{array}$ & $\begin{array}{c}49,15 \% \\
(42,74-55,56)\end{array}$ & NS \\
\hline HTA con RCV añadido y con estatina & $\begin{array}{c}36,6 \% \\
(21,03-52,24)\end{array}$ & $\begin{array}{c}37,0 \% \\
(23,58-50,49)\end{array}$ & $\begin{array}{c}36,86 \% \\
(26,68-47,05)\end{array}$ & $\begin{array}{l}\text { NS } \\
0,06\end{array}$ \\
\hline HTA con RCV con estatina y LDL $<130 \mathrm{mg}$ & $\begin{array}{c}70,27 \% \\
(55,47-85,07)\end{array}$ & $\begin{array}{c}54,0 \% \\
(40,11-67,89)\end{array}$ & $\begin{array}{c}60,92 \\
(50,61-71,22)\end{array}$ & \\
\hline HTA + DM con Estatina con Antiagregante & $\begin{array}{c}36,36 \% \\
(9,01-63,72)\end{array}$ & $\begin{array}{c}28,89 \% \\
(4,12-53,65)\end{array}$ & $\begin{array}{c}32,05 \% \\
(13,66-50,44)\end{array}$ & NS \\
\hline HTA + DM con control metabólico adecuado & $\begin{array}{c}30,3 \% \\
(1,67-58,93)\end{array}$ & $\begin{array}{c}40 \% \\
(17,25-62,75)\end{array}$ & $\begin{array}{c}35,9 \% \\
(18,04-53,76)\end{array}$ & NS \\
\hline
\end{tabular}

Tabla 2. Objetivos del estudio: cumplimiento de recomendaciones. 
por lo que podrían haber escapado del numerador, infraestimando la prevalencia de HTA y sobreestimando la proporción de pacientes con cifras controladas.

- Un estudio paralelo de calidad de los registros en la historia clínica informatizada de nuestro Equipo de Atención Primaria ha mostrado problemas de infraregistro en datos de laboratorio, variables antropométricas, e incluso de cifras de presión arterial en consulta. Ello ha podido determinar que se excluyan en este análisis historias de pacientes hipertensos, redundando en una sobreestimación de pacientes controlados (probablemente los pacientes controlados son los que más registros tienen, pudiendo existir asociación entre calidad de registro y calidad del control).

- La proporción de pacientes hipertensos tratados con los modernos y eficaces ARA II, solos o en combinación, es muy elevada (muy próxima al 50\%) lo que podría también contribuir a una mayor proporción de pacientes controlados en comparación con los datos publicados de otros estudios.

El grado de adherencia de los profesionales del EAP Manzanares II a la Guía SEH/SEC 2007 es variable, pero en cualquier caso bajo, desconociéndose si es por no utilizar las Guías, como sugieren otros estudios3, o por no adecuar las actuaciones terapéuticas a la realidad (inercia terapéutica), ya que:

- Poco más de uno de cada tres hipertensos con riesgo CV añadido tiene prescrita estatina. De ellos, dos de cada tres tratados alcanzan el nivel objetivo de colesterol LDL, lo que representa sólo dos de cada nueve hipertensos con riesgo CV añadido.

- Apenas uno de cada tres hipertensos diabéticos tiene prescrito tratamiento antiagregante + estatina, y de ellos sólo cuatro de cada diez alcanzan objetivo de colesterol LDL, lo que representa apenas algo más que uno de cada diez hipertensos diabéticos.

Como conclusión, el porcentaje de pacientes hipertensos controlados es elevado en relación con los datos publicados en nuestro ámbito, lo que, expresado con reservas, creemos es un buen dato de calidad asistencial, a refrendar en ulteriores estudios una vez extendido el uso de la Guía SEH/SEH y mejorada la calidad de los registros de la historia clínica informatizada por los profesionales de nuestro Equipo.

El cumplimiento de las recomendaciones de la Guía SEH/SEC 2007 de HTA en el Centro de Salud Manzanares II es bajo. Creemos que es necesaria una actualización de los profesionales médicos y de enfermería para su conocimiento e implementación, en aras de conseguir un abordaje integral, así como un mayor grado de calidad y adecuación en la atención prestada.

\section{BIBLIOGRAFÍA}

1. Mancia G, De Baker G, Dominiczak A, Cifkova R, Fagard R, Germano G et al. 2007 ESH-ESC Practice Guidelines for the Management of Arterial Hypertension: ESH-ESC Task Force on the Management of Arterial Hypertension. J Hypertens 2007; 25(9):1751-62.

2. Bramlage P, Thoenes M, Kirch W, Lenfant C. Clinical practice and recent recommendations in hypertension management-reporting a gap in a global survey of 1259 primary care physicians in 17 countries. Curr Med Res Opin 2007; 23(4):783-91.

3. González-Juanatey JR, Alegria-Ezquerra E, Aznar-Costa J, Bertomeu-Martínez V, Franch-Nadal J, Palma-Gamizf JL. Knowledge and implementation of cardiovascular risk clinical practice guidelines by general practitioners and specialists.Rev Esp Cardiol 2006; 59(8):801-6.

4. Burgers JS, Simmoons ML, Hoest AW, Stehouwer CD, Staman WA. Guideline "Cardiovascular Risk Management".Ned Tijdschr Geneeskd 2007; 12; 151(19):1068-74.

5. Grover SA, Hemmelgarn B, Joseph L, Milot A, Tremblay G.. The role of global risk assessment in hypertension therapy. Can $J$ Cardiol 2006; 22(7):606-13.

6. Smith EE, Abdulah AR, Amirfazan H, Schwamm LH. Serum lipid profile on admission for ischemic stroke failure to meet National Cholesterol Education Program Adult Treatment Panel (ATPIII) guidelines. Neurology 2007; 68(9):660-5.

7. Cannon CP Steinberg BA, Murphy SA, Mega JL, Braunwald E. Meta-Analisis of cardiovascular outcomes trials comparing intesive versus moderate statin therapy. J Am Coll Cardiol 2006; 48(3):438-45.

8. Lobos JM, Castellanos A. Grupo de trabajo de Enfermedad Cardiovascular de la semFYC. Actitud frente a un paciente con factores de riesgo cardiovascular. C\&AP 2006; 3:107-114.

9. Botey Puig A, Coca Payeras A, de la Sierra Iserte A, Ferreira Montero IJ. Hipertensión arterial y cardiopatia hipertensiva. En: Farreras Valentí P, Rozman C. Medicina Interna. XV ed. Madrid: Elsevier; 2005. p. 587-611.

10. De la Figuera von Wichmann M, Dalfó i Baiqué A. Hipertensión Arterial. En: Martín Zurro A, Cano Pérez JF. Atención Primaria. Conceptos, organización y práctica clínica. V ed. Madrid: Elsevier; 2003. p. 764-798.

11. Vilaseca Canals J, Maiques Galán A. Dislipemias. Riesgo cardiovascular. En: Martín Zurro A, Cano Pérez JF. Atención Primaria. Conceptos, organización y práctica clínica. V ed. Madrid: Elsevier; 2003. p. 799-820.

12. Rodríguez-Coca GC, Alonso-Moreno FJ, Barrios V, Llisterri JL, Lou S;Matalí A, et al. Características de la presión arterial en una población dislipémica española asistida en Atención Primaria Estudio LIPICAPPA. Rev Esp Cardiol 2007; 60:825-832.

13. Alvarez- Sala LA, Suárez C, Mantilla T, Franch J, Ruilope LM, Banegas JR et al. Estudio PREVENCAT: control del riesgo cardiovascular en atención primaria. Med Clín (Barc) 2005; 124(11):406-410.

14. Llisterri JL, Rodríguez GC, Alonso FJ, Lou S, Divisón JA, Santos $\mathrm{JA}$, et al. Control de la presión Arterial en la población hipertensa española atendida en atención primaria. Estudio PRESCAP 2002. Med Clin (Barc) 2004; 122(5):165-171.

15. Grupo ELIPSE. Efectividad en el control de factores de riesgo cardiovascular en diabéticos tipo 2 de la provincia de Ciudad Real. Rev Clin Esp 2005; 205(5):218-222. 